Joachim Wiemeyer ${ }^{1}$

Ruhr-Universität Bochum

\title{
Jugendarbeitslosigkeit - eine große sozialethische Herausforderung in Europa
}

„Zu einem besonderen Übelstand wird sie (die Arbeitslosigkeit J.W.), wenn sie vor allem Jugendliche trifft, die nach einer entsprechenden allgemeinbildenden, technischen und beruflichen Vorbereitung keinen Arbeitsplatz finden können und ihren ehrlichen Arbeitswillen verbunden mit ihrer Bereitschaft, die ihnen zukommende Verantwortung für die wirtschaftliche und soziale Entwicklung der Gesellschaft zu übernehmen, schmerzlich enttäuscht sehen." Diese Besorgnis Johannes Paul II. in der Enzyklika Laborem Exercens (Nr. 18) aus dem Jahr 1981 stellt heute eine bittere Realität in vielen Ländern Europas dar.

Im Gefolge der Finanz- und der anschließenden Staatsschuldenund Eurokrise ist die Arbeitslosigkeit von Jugendlichen unter 25 Jahren auf etwa 5 Millionen in der gesamten EU gestiegen. Hinzukommen 2,8 Mill. junge Menschen, die sich weder in Ausbildung, noch in Arbeit befinden, sondern sich vermutlich vielfach resigniert in den privaten Haushalt der Eltern oder Ehepartner zurückgezogen haben. Für eini-

1 Prof. f. Christliche Gesellschaftslehre an der Kath.- Theol. Fakultät der RuhrUniversität Bochum, Dr. rer.pol, (Volkswirtschaftslehre), lic. theol., Habilitation in Christliche Sozialwissenschaften, Berater der Caritaskommission der Deutschen Bischofskonferenz. Forschungsschwerpunkte: Politische Ethik, Wirtschaftsethik, Wohlfahrtsverbände und Sozialpolitik. E-mail: joachim.wiemeyer@rub.de. 
ge Länder werden Arbeitslosenquoten von mehr als 50\% angegeben. Während in Deutschland die Jugendarbeitslosigkeit von 2007-2014 um 4,1 Prozentpunkte sogar sank, stieg sie in Ländern wie Spanien, Zypern, Italien, Kroatien und Griechenland um 20-35 Prozentpunkte an. Die EU beschloss daraufhin 2013 ein Programm in Höhe von 6 Mrd. zur Bekämpfung der Jugendarbeitslosigkeit. Diese "Jugendgarantie“ zielte darauf ab, dass kein Jugendlicher länger als vier Monate ohne das Angebot einer Arbeitsstelle, eines Praktikums oder einer Weiterbildung bleiben sollte. Es flossen aber nur wenige Mittel ab.

Wenn der Start ins Erwerbsleben für viele von vornherein nicht gelingt, kann dies für die gesamte Erwerbsbiographie erhebliche Folgen haben, weil man nur eine geringer qualifizierte Tätigkeit erhält und auch später in der Berufslaufbahn häufiger arbeitslos wird. Ebenso hat dies Konsequenzen für das Vertrauen in die gesellschaftliche Ordnung.

Im Folgenden wird in einem ersten Schritt die Problematik der Jugendarbeitslosigkeit geschildert. Weiterhin werden Ursachen und Folgen hoher Jugendarbeitslosigkeit aufgezeigt. Da eine hohe Jugendarbeitslosigkeit, vor allem wenn sie bedeutend größer als die Arbeitslosigkeit von Erwachsenen ist, erhebliche Fragen der Gerechtigkeit zwischen Generationen aufwirft, erfolgt im zweiten Schritt eine sozialethische Reflexion. Im dritten Schritt sind dann Handlungswege aufzuzeigen, wie Arbeitslosigkeit von Jugendlichen reduziert werden kann.

\section{Jugendarbeitslosigkeit: Ursachen und Folgen}

Die Arbeitslosigkeit von Jugendlichen zwischen 15 und 25 Jahren in Europa ist verschieden groß. Während sie 2014 in Deutschland lediglich $7,7 \%$ betrug, belief sie sich in Polen auf $23,9 \%$, ihr Höchststand lag in den EU-Ländern Griechenland und Spanien bei 52,4\%, bzw. 53,2\%. Auch der Abschluss eines Hochschulstudiums reduzierte in den Krisenländern die Arbeitslosenquote kaum. Sie lag in Griechenland bei 40,6\%, in Spanien bei $28,1 \%$ und in Italien bei $26,8 \% .^{2}$

2 Vgl. G. Bosch, Jugendarbeitslosigkeit in Europa-warum versagen milliardenschwere Hilfsprogramme?, „Ifo-Schnelldienst“ 68 (2015) 17, S. 7-11, hier S. 9. 
„Die Jugend im Religionsunterricht ist auch nicht mehr die, die sie mal war"...

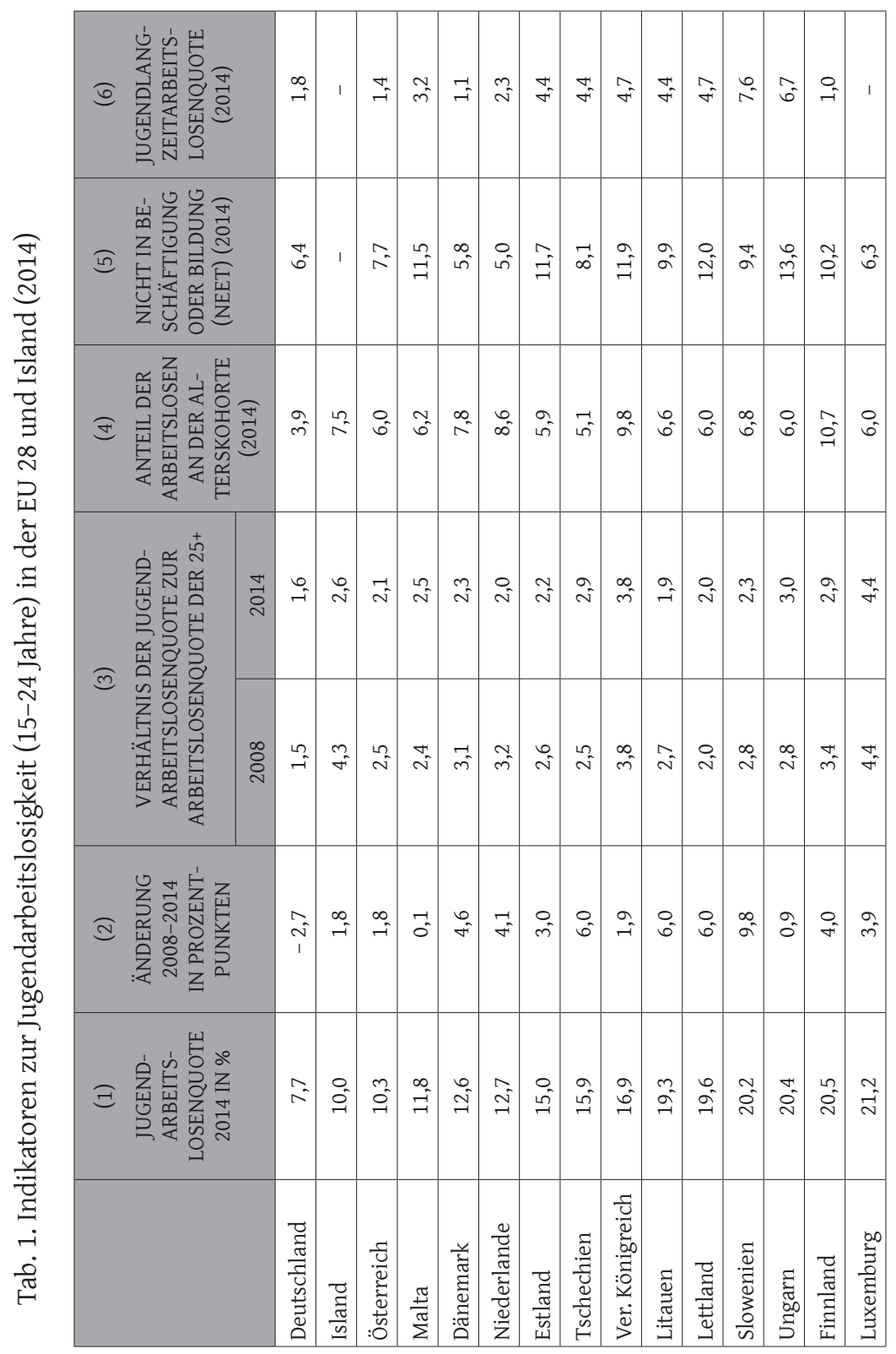




\section{Joachim Wiemeyer}

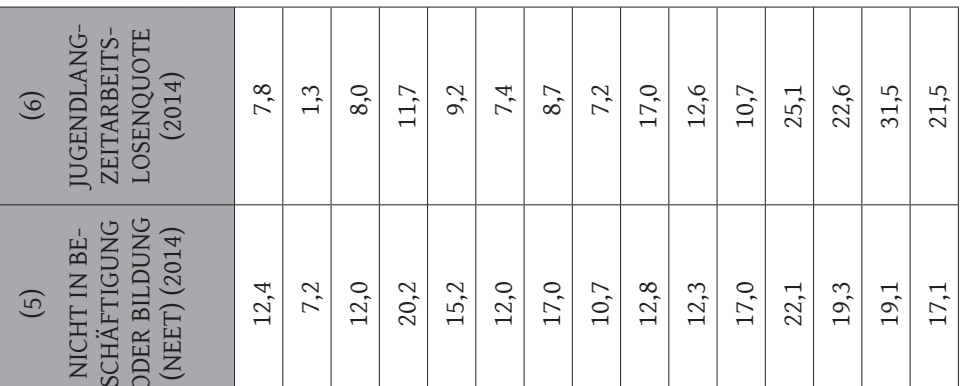

嵒峑崖

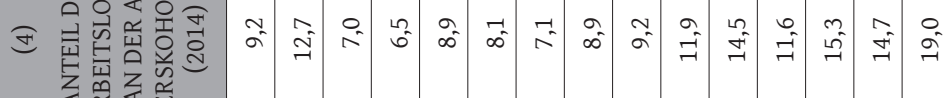

《余《嵌

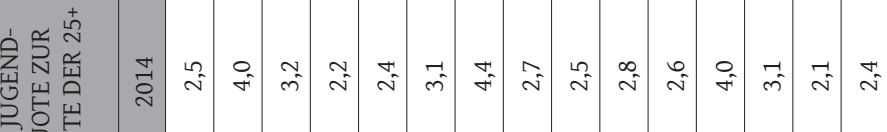

बิ

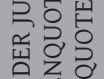

岸峞岂

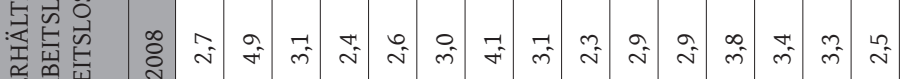

它

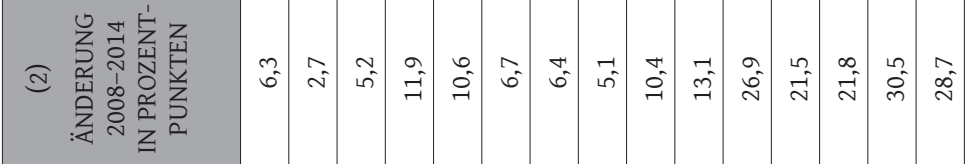

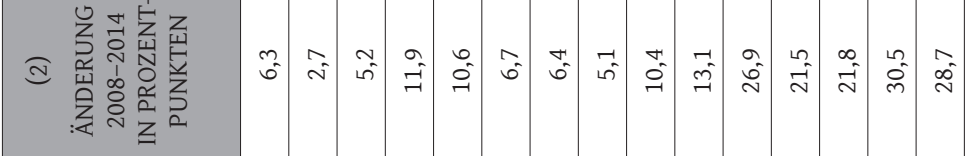

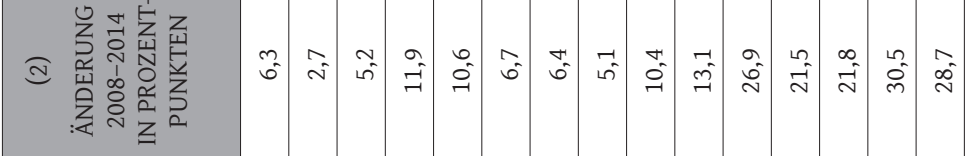

覓

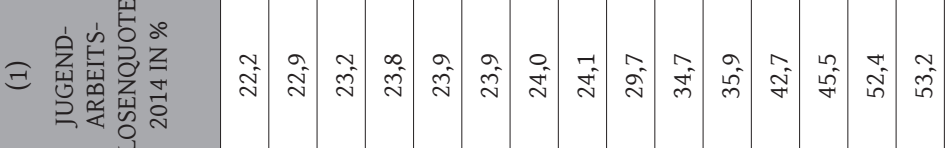


Wenn Länder eine hohe Arbeitslosenquote bei Jugendlichen vorweisen, kann dies zweierlei bedeuten: Erstens gibt es eine generell hohe Arbeitslosigkeit, die auch Erwachsene betrifft. Zweitens kann die Arbeitslosigkeit bei Jugendlichen besonders hoch sein, so dass sie benachteiligt werden. Während in Deutschland die Arbeitslosenquote bei Jugendlichen lediglich das 1,6fache der Arbeitslosenquote für über 25-Jährige betrug, im EU-Durchschnitt das 2,5fache, übertraf sie in Polen das 3,1-, in Italien sogar das 4,4-Fache den EU-Durchschnitt deutlich. In Griechenland ist sie seit Beginn der Wirtschaftskrise vom 3,3- auf das 2,1-Fache sogar gesunken, weil die allgemeine Arbeitslosigkeit so stark gestiegen war.

Eine Betrachtung von Arbeitslosenquoten greift aber zu kurz. Unter 25 Jahren besuchen viele Jugendliche noch Schulen und Universitäten. Daher gibt die Jugendarbeitslosigkeit das Verhältnis der Jugendlichen, die bereits auf dem Arbeitsmarkt sind, zu denjenigen wieder, die arbeitslos sind. Wenn man die Arbeitslosenquoten in Beziehung zum gesamten Jahrgang setzt, reduzieren sich die Arbeitslosenquoten deutlich. In Deutschland beträgt sie lediglich 3,9\%, in Polen $8,1 \%$, in Griechenland $14,7 \%$ und Spanien $19 \%$.

Allerdings muss man auch beachten, dass es Jugendliche gibt, die weder einer schulischen oder universitären Ausbildung nachgehen, noch in Arbeit sind oder sich arbeitslos gemeldet haben. Zwar sind manche von ihnen als Mutter im eigenen Haushalt beschäftigt, andere haben sich aber resigniert vom Arbeitsmarkt zurückgezogen. Wenn man diese NEET- (Not in Education, Employment or Training: nicht in Bildung, nicht in Beschäftigung) Quote hinzunimmt, kommt man zu anderen Daten. In Deutschland beträgt diese Quote 6,4\%, im EU- Durchschnitt 12,4\%, in Polen $12 \%$, in Italien 22,1\%, in Griechenland 19,1\% und in Spanien $17,1 \%$.

Die Arbeitslosigkeit von Jugendlichen ist in allen Ländern im Vergleich zu Erwachsenen erstens deshalb höher ${ }^{3}$, weil es - auch bei guter Beschäftigungslage - kurze Zeiten von Übergangsarbeitslosigkeit nach der Schule, nach einer Ausbildung, nach einem Studium oder der Ableistung

3 Vgl.J. Möller, Jugendarbeitslosigkeit in Europa:Generation ohne Zukunft, „Ifo-Schnelldienst“ 68 (2015) 17, S. 3-6. 
von Wehrdienst geben kann. Zweitens verfügen Jugendliche üblicherweise nicht die berufs- und aufgabenbezogen Fähigkeiten, die bereits in einem Betrieb Beschäftigte besitzen. Drittens gilt häufig bei Entlassungen, dass diejenigen, die zuletzt gekommen sind, zuerst wieder gehen müssen. Dabei spielen auch soziale Faktoren eine Rolle, weil Ledige eher entlassen werden als Väter oder Mütter, die für Kinder sorgen müssen. Viertens sind viele Jugendliche noch auf der Suche nach einem Beruf, der ihnen gefällt, so dass sie eher kündigen, um möglicherweise auch an einem anderen Ort eine andere Tätigkeit auszuprobieren.

Jugendarbeitslosigkeit kann in Ländern hoch sein, in denen es ein starkes Bevölkerungswachstum gibt. Dies ist aber in den meisten EU-Ländern etwa im Gegensatz zu Ländern Nordafrikas - nicht der Fall. ${ }^{4}$ So gehören Griechenland (10,4\%), Italien (9,7\%) und Spanien (9,8\%) (sowie Slowenien) zu den vier EU-Ländern, die den geringsten Anteil von 15-24 Jährigen an der Bevölkerung haben. In Polen liegt dieser Anteil bei 12,4\%, in Deutschland 10,8\%. In einigen Ländern läge die Arbeitslosenquote noch deutlich höher, wenn es nicht wie im Fall Polen durch unfreiwillige Auswanderung eine gewisse Entlastung des heimischen Arbeitsmarktes geben würde. Die Hälfte der rd. 2 Millionen sind zwischen 20-29 Jahre alt. ${ }^{5}$

Diese Gesichtspunkte relativieren zwar die Arbeitslosigkeit von Jugendlichen. Umgekehrt muss man aber bedenken, dass es Faktoren gibt, die Jugendarbeitslosigkeit bzw. die Arbeitsbedingungen beschäftigter Jugendlicher unterschätzen. Es kann sein, dass Jugendliche länger an der Universität verbleiben, weil sie im Moment keine Arbeitsmarktchancen sehen. ${ }^{6}$ Andere haben zwar eine Tätigkeit erhalten, sind aber, obwohl sie ein Studium abgeschlossen haben, lediglich in einer niedrigqualifizierten Tätigkeit beschäftigt. Auch ist ihre Bezahlung gering und ihr Beschäftigungsverhältnis prekär, da sie nur über einen zeitlich befristeten Vertrag verfügen oder gegen ihren Wunsch nur in Teilzeit beschäftigt sind.

4 Vgl. G. Bosch, Jugendarbeitslosigkeit in Europa - warum versagen milliardenschwere Hilfsprogramme?, „Ifo-Schnelldienst“ 68 (2015) 17, S. 9.

5 Vgl. M. Polakowski, Youth Unemployment in Poland, November 2012 (Friedrich-EbertStiftung), S. 9.

6 Vgl. G. Bosch, Jugendarbeitslosigkeit in Europa - warum versagen milliardenschwere Hilfsprogramme?, „Ifo-Schnelldienst“ 68 (2015) 17, S. 7. 
Ein hohes Ausmaß von Jugendarbeitslosigkeit kann zum einen konjunkturelle Ursachen haben. Dies gilt dann, wenn auch die Arbeitslosigkeit von Erwachsenen sehr hoch ist. Wenn die Jugendarbeitslosigkeit im Verhältnis zur Arbeitslosigkeit Erwachsener besonders hoch ist, liegen aber strukturelle Gründe vor. ${ }^{7}$ Ein erster struktureller Grund liegt in einem Schulsystem, das eine hohe Zahl von Absolventen hervorbringt, die keinen Schulabschluss erreichen. Ein zweiter Grund liegt darin, dass Formen der schulischen und universitären Ausbildung nicht den Anforderungen des Arbeitsmarktes entsprechen, so dass viele Jugendliche fehlqualifiziert sind. Ein dritter Grund liegt darin, dass neu auf den Arbeitsmarkt tretende Jugendliche Outsider sind. Insider sind diejenigen Beschäftigten, die bereits über einen Arbeitsplatz verfügen und ihr Lohnniveau und ihre gesicherte Stellung verteidigen wollen, aber auch für die Jugendlichen keine soziale Verantwortung verspüren, weil diese theoretisch nicht nur in ihrem Betrieb, sondern überall einen Arbeitsplatz erhalten könnten.

Die deutschsprachigen Länder (Deutschland, Österreich, Schweiz) weisen eine im globalen Wettbewerb leistungsfähige Wirtschaft, nur geringe Akademikerquoten, aber zugleich eine geringe Jugendarbeitslosigkeit ${ }^{8}$ auf. Das duale Ausbildungssystem, nämlich eine überwiegende praktische Ausbildung im Betrieb ergänzt durch die Berufsschule sowie ein duales Studium, in Kombination von Arbeitsstelle und Betrieb, hat drei zentrale Vorteile: Die Betriebe haben in die Ausbildung der Jugendlichen investiert und werden auch zumindest einen Teil der Fertigausgebildeten übernehmen. Durch ihre Ausbildung sind sie für Gewerkschaften, Betriebsräte sowie die gesamte Belegschaft zu Insidern geworden. ${ }^{9}$ Daher wird z.B. in einer Wirtschaftskrise die gesamte Belegschaft über Kurzarbeit das verminder-

7 Vgl.J. Möller, Jugendarbeitslosigkeit in Europa:Generation ohne Zukunft, „Ifo-Schnelldienst“ 68 (2015) 17, S. 3f.

8 Vgl. G. Bosch, Jugendarbeitslosigkeit in Europa - warum versagen milliardenschwere Hilfsprogramme? "Ifo-Schnelldienst“ 68 (2015) 17, S. 9; F. Rauner, Was tun gegen die Jugendarbeitslosigkeit in Europa? Einführung der dualen Berufsausbildung, „Ifo-Schnelldienst" 66 (2013) 16, S. 18-21.

9 Vgl. G. Bosch, Was tun gegen die Jugendarbeitslosigkeit in Europa?, „Ifo-Schnelldienst" 66 (2013) 16, S. 6-10. 
te Arbeitsvolumen mit Einkommensverzichten tragen. Möglicherweise wird die Unternehmensleitung mit Betriebsrat und Gewerkschaften vereinbaren, dass einige ältere Arbeitnehmer (auch Väter und Mütter im Interesse ihrer Töchter und Söhne) vorzeitig in den Ruhestand eintreten, um Jugendlichen den Eintritt in den Arbeitsmarkt zu ermöglichen. Selbst in der schweren Wirtschaftskrise 2009 traten in Deutschland über 500000 Jugendliche in eine berufliche Ausbildung ein und wurden so in den Arbeitsmarkt integriert.

Folgen hoher Jugendarbeitslosigkeit sind, dass bei längerer Arbeitslosigkeit möglicherweise Fähigkeiten, die in einer Ausbildung bzw. in einem Studium gerade erst erworben wurden, wieder verloren gehen. So kann sich Jugendarbeitslosigkeit langfristig negativ auf ein Arbeitsleben auswirken, weil man dauerhaft keinen Arbeitsplatz erhält, der der formalen Qualifikation entspricht. Weiterhin kann kein hinreichendes Selbstvertrauen aufgebaut und ein optimistisches Herangehen an Herausforderungen erst gar nicht entstehen. Gerade Schwächere können auch im Verlauf ihrer Erwerbsphase eine schwierig verlaufende Integration in die Erwerbsarbeit nur schwer kompensieren. ${ }^{10}$ In der zwangsweise erweiterten Freizeit kommt es dazu, dass verstärkt Alkohol oder andere Drogen konsumiert werden. ${ }^{11}$ Auch kann abweichendes Verhalten (z. B. Diebstahl) auftreten. Zudem werden die niedrigen Geburtenraten in manchen europäischen Ländern auf Jugendarbeitslosigkeit bzw. prekäre Arbeitsverhältnisse zurückgeführt. ${ }^{12}$ Diese Mutmaßung trifft aber auf Deutschland nicht zu, weil dort die Geburtenzahlen in einer Zeit der Überbeschäftigung (mehr freie Stellen als Arbeitslosen, in Jahren, in denen mehrere 100000 ausländische Arbeitskräfte angeworben wurden) bereits Anfang der 70er Jahre zurückgingen. Eine weitere Folge besteht darin, dass junge Menschen Vertrauen in das Wirtschaftssystem und das politische System ihres Landes sowie der europäischen Union verlieren. Dies kann zur Wahl popu-

10 Vgl. J. Möller, Jugendarbeitslosigkeit in Europa: Generation ohne Zukunft, „Ifo-Schnelldienst" 68 (2015) 17, S. 3.

11 Vgl. D. Tamesberger, Jugendarbeitslosigkeit in Europa: Eine Beschreibung des Problemausmaßes und der Folgen, „Wiso“ 1 (2014), S. 139-158.

12 Vgl. K. D. Sohn, S. Czuratis, Jugendarbeitslosigkeit in den Mitgliedstaaten der EU - was kann und sollte die EU tun?, „Ifo-Schnelldienst“ 66 (2013) 16, S. 14--7, hier 15. 
listischer Parteien führen. Arbeitslose Jugendliche nehmen weniger als Schüler und Studenten sowie Erwerbstätige am gesellschaftlichen Leben (Mitgliedschaft in Parteien, Vereinen, Verbänden) teil.

\section{Jugendarbeitslosigkeit eine Frage der Generationengerechtigkeit}

Eine gerechte gesellschaftliche Ordnung stellt auch im Verhältnis verschiedener Alterskohorten bzw. Generationen Anforderungen. ${ }^{13}$ So strebt die ältere Generation an, kulturelle, ethische und religiöse Werte an die jüngere Generation weiterzugeben. Dabei kann es zu Generationenkonflikten kommen, die in Deutschland aber relativ gering sind. Auf der materiellen Ebene haben Großeltern und Eltern vielfach den Wunsch „Unseren Kindern bzw. Enkeln soll es in Zukunft besser gehen. “ Daher unterstützen Eltern und Großeltern ihre Kinder und Enkel vielfach finanziell, soweit ihnen dies möglich ist. Dies geschieht nicht nur, weil sie sie selbst erwarten von diesen im Alter versorgt und gepflegt zu werden.

Das Verhältnis der Generationen betrifft weitere Bereiche: Das Sachkapital der Gesellschaft (Häuser, Infrastruktur etc.) ist zu erhalten und auszubauen. Vielfach hatten die sozialistischen Volkswirtschaften des Ostblocks vor 1990 aufgrund der wenig effizienten Wirtschaftsform zu wenig in den Erhalt der Infrastruktur, der Gebäude und Fabrikanlagen investiert. Weiterhin betrifft dies die natürliche Umwelt. Hier soll möglichst das natürliche Kapital einer Gesellschaft erhalten bleiben, indem z. B. nur so viel Holz geschlagen wird, wie auf natürliche Weise nachwächst. Außerdem dürfen nicht so viele Schadstoffe (z. B. Müll) an die Umwelt abgegeben werden, dass neuen Generationen ein schlechte-

13 Vgl. zur Generationengerechtigkeit:J. Wiemeyer, Gerechtigkeit zwischen Generationen als wirtschaftsethisches Problem, „Ethica“ 12 (2004) 1, S. 71-94; ders., Die Solidarischen Verpflichtungen der älteren Generation gegenüber Kindern und Jugendlichen, [in:] Intergenerationalität zwischen Solidarität und Gerechtigkeit, Hg. J. Eurich, P. Dabrock, W. Maaser, Heidelberg 2008, S. 133147; ders., Sprawiedliwość międzypokoleniowa jako wyzwanie społeczno-etyczne, „Roczniki Nauk Społecznych" (2011) 3 (39), S. 69-87; ders., Alterssicherung und Politik bei schrumpfender Bevölkerung, „Polonia Sacra“ 19 (2015) Nr 1 (38), S. 5-26. 
rer Naturzustand hinterlassen wird oder durch einen Klimawandel die Lebensbedingungen grundlegend verschlechtert werden.

Eine weitere Verantwortung der älteren Generation betrifft die Größe der Generationen selbst. Wenn es - wie in Entwicklungsländern - eine zu große Anzahl von Kindern gibt, kann es bei begrenzter Landfläche immer schwieriger werden, diese zu ernähren, eine gute Schulbildung zu gewährleisten, so dass die nachfolgenden Generationen keine besseren Lebensbedingungen vorfinden. Umgekehrt darf eine nachfolgende Generation nicht so klein werden, dass es in Zukunft schwierig wird, die Alterssicherung der älteren Generation zu gewährleisten. Man kann nicht erwarten, dass man selbst lediglich 15-20\% seines Einkommens für die Versorgung der eigenen Eltern aufwenden muss, während man von der jüngeren Generation erwartet, dass diese 25-30\% ihres Einkommens dafür bereitstellt.

Für die Chancen junger Menschen in der gegenwärtigen- durch Globalisierung und digitale Technik bestimmte Welt - ist der Wissenserwerb (Humankapital) zentral. Hinter diesem stehen das erwähnte Sach- und Naturkapital zurück. Daher besteht ein zentrales Interesse von jungen Menschen darin, eine gute schulische Ausbildung zu erhalten. Dazu ist ein gutes Schulsystem, das jedem Schüler ermöglicht, seine Begabungen zu entfalten, wichtig. Die Inhalte des Unterrichts, die Ausstattung der Schulen mit technischem Gerät usw. müssen den einzelnen Schüler eine Bildung ermöglichen, die sie oder ihn befähigt zu einem selbstständigen und verantwortungsbewussten Bürger seines Gemeinwesens heranzureifen. Dies beinhaltet neben kulturellen, religiösen und politischem Wissen auch beruflich einsetzbare Fähigkeiten. Nach der Schulbildung sollte eine berufsbezogene Ausbildung oder ein Studium offenstehen. Dabei muss diese Ausbildung einen effektiven Zugang zum Arbeitsmarkt ermöglichen. Solche Bildungsmöglichkeiten sind im ganzen Land anzubieten, so dass auch regional nicht zu ungleiche Bildungschancen bestehen, wenn gleich die Fülle des Bildungsangebots von Großstädten und Metropolen nicht überall möglich ist.

Wenn eine konjunkturell schwierige Situation besteht, ist es Aufgabe der allgemeinen Wirtschafts-, Finanz- und Geldpolitik für einen hohen Beschäftigungsstand zu sorgen. Lasten eines konjunkturellen Einbruchs 
dürfen nicht einseitig eine bestimmte Alterskohorte der Bevölkerung treffen, sondern müssen breiter gestreut werden, so dass alle diese Lasten und Einbußen tragen müssen.

In vielen europäischen Ländern gab es bereits vor der Euro- und Finanzkrise eine hohe strukturelle Jugendarbeitslosigkeit. Eine solche strukturelle Jugendarbeitslosigkeit widerspricht zum einen den Gedanken der Generationengerechtigkeit, zum anderen stellt sie aber auch einen Schaden für das Gemeinwohl dar, wenn man gesunde - und häufig qualifizierte - junge Menschen in einer besonders leistungsfähigen Lebensphase vom Einsatz ihrer produktiven Kräfte fernhält. Es ist daher geboten, solche Regelungen und Institutionen auf dem Arbeitsmarkt und den Betrieben zu finden, die hohe Arbeitslosigkeit vermeiden.

Natürlich haben auch Jugendliche Verpflichtungen, etwa in Schule, Hochschule und beruflicher Ausbildung zu lernen und die notwendige Disziplin und ein hinreichendes Durchhaltevermögen aufzuweisen. Nicht jeder Jugendliche kann seinen Lieblingsberuf ergreifen, an seiner Lieblingsuniversität sein Lieblingsfach studieren oder an seinem Lieblingsort einen Arbeitsplatz finden. Ebenso wie bei der Bezahlung wird man gewisse Abstriche machen müssen, um sich gesellschaftlich einzubringen.

\section{Wege aus der Jugendarbeitslosigkeit}

\subsection{Kurzfristig}

Um die Jugendarbeitslosigkeit in vielen EU-Ländern zu reduzieren, gibt es zwei zeitliche Dimensionen: Die erste betrifft kurzfristig die gegenwärtig hohe Jugendarbeitslosigkeit. Dazu wurde auf dem EU-Gipfel 2013 ein 6,4 Milliarden-Euro-Programm beschlossen. Dieses Programm sollte ermöglichen, dass Jugendliche nicht länger als 4 Monate arbeitslos sind und dann ein Angebot eines Praktikums, einer betrieblichen Ausbildung, eines Auslandsaufenthalts etc. erhalten. Dieses Programm wurde sehr schleppend umgesetzt. Wenn in Italien, Spanien und Griechenland die Arbeitsverwaltung so schlecht organisiert ist, dass auf über 200 Arbeitslose 
nur ein Sachbearbeiter kommt ${ }^{14}$, ist dieser angesichts einer so großen Zahl gar nicht in der Lage, sich um alle Jugendlichen zu kümmern. In Deutschland sind es nur 26, so dass sich ein Mitarbeiter der Arbeitsverwaltung viel intensiver mit einem arbeitslosen Jugendlichen beschäftigen kann, um seine Fähigkeiten und Neigungen aufzuspüren und ihn zur Arbeit zu motivieren.

In der EU gibt es solche Teufelskreise, die auch aus der Entwicklungszusammenarbeit der Dritten Welt bekannt sind: Wenn bestimmte Institutionen der Gesellschaft nicht funktionieren, ist ein Land oder eine Region unterentwickelt. Wenn man diesen dann finanzielle Ressourcen zur Verfügung stellt, ist man entweder nicht in der Lage, die Mittel sachgerecht und effizient auszugeben, so dass sie gar nicht abgerufen werden oder die Mittel werden vergeudet oder unterschlagen.

Ein anderer kurzfristig angezielter Weg besteht darin, dass Jugendliche aus Ländern mit hoher Arbeitslosigkeit in Länder wie Deutschland auswandern, in denen es freie Ausbildungsplätze und freie Arbeitsplätze gibt. ${ }^{15}$ Dies ist in den letzten Jahren geschehen, indem besonders viele junge Menschen aus Südeuropa nach Deutschland gekommen sind. Dieser Prozess verlief aber nicht problemlos, weil sprachliche Anforderungen, andere klimatische Bedingungen und Lebensformen sowie die Trennung von Familie und Freunde Hemmnisse darstellen, so dass ein Teil - trotz der dortigen prekären Beschäftigungslage - wieder in die Heimat zurückgekehrt ist und auf einen sicheren und relativ gutbezahlten Arbeitsplatz in Deutschland verzichtet wurde. Auch aus Polen sind viele Jugendliche migriert. Es sollen lediglich 10\% der polnischen Hochschulabsolventen im Ausland eine Beschäftigung entsprechend ihrer formalen Qualifikation erhalten, während andere Hochschulabsolventen eher niedrigqualifizierte Tätigkeiten ausüben. Daher kommen hier ein „brain drain“ und ein „brain waste“ zusammen. ${ }^{16}$ Dies könnte durch eine stärker gesteuerte Wanderungspolitik vermieden werden, wenn es Abkommen zwi-

14 Vgl. Gerhard Boch, Jugendarbeitslosigkeit in Europa - warum versagen milliardenschwere Hilfsprogramme?, „Ifo-Schnelldienst“ 68 (2015) 17, S. 11.

15 Dies wird von W. Eichhorst, H. Hinte, U. Rinne, Jugendarbeitslosigkeit in Europa: Status Quo und (keine?) Perspektiven, IZA Standpunkte Nr. 57, S. 8f. befürwortet.

16 Vgl. M. Polakowski, Youth Unemployment in Poland, op. cit., S. 9. 
schen den Staaten geben würde, die z. B. eine qualifikationsgerechte Arbeitsplatzvermittlung durch richtige Vorbereitung (z. B. Sprachkurse) ermöglichen.

Langfristig stellen solche Arbeitskräftewanderungen keine Lösung dar. Praktisch alle EU-Länder weisen eine niedrige Geburtenrate auf. Wenn aus einer jüngeren Generation noch besonders qualifizierte, risikobereite und initiative Personen dauerhaft auswandern, kann ein Land nicht prosperieren. Kurzfristige Vorteile für Länder wie Deutschland, die junge und hoch motivierte Arbeitskräfte erhalten, wirken sich langfristig negativ aus, weil andere EU-Länder zurückfallen werden, so dass die Spannungen in der EURO-Zone zunehmen, diese Länder verstärkt nach EU-weiten Transfers rufen werden und die kurzfristigen ökonomischen Vorteile für Deutschland auf Dauer zu zusätzlichen Zahllasten in den EU-Haushalt führen.

Nur wenn es den Heimatländern der Migranten gelingt, einen wirtschaftlichen Aufschwung zu erreichen, so dass zumindest ein Teil der Migranten mit neuen Qualifikationen (z. B. deutschen Sprachkenntnissen sowie einer Ausbildung und beruflicher Praxis in Deutschland) zurückkehrt, können Wanderungsbewegungen eine kurzfristige partielle Problemlösung sein.

Zur Milderung der Jugendarbeitslosigkeit in konjunktureller Hinsicht könnte beitragen, wenn es gelingen sollte, einen wirtschaftlichen Aufschwung herbeizuführen. Dazu gehört, dass z. B. Altlasten aus der Finanzkrise im Bankensektor beseitigt werden. Weiterhin muss die Finanz-, Wirtschaftsund Sozialpolitik einen Aufschwung ermöglichen.

Weiterhin ist die Förderung der Selbstständigkeit durch Kreditprogramme für Existenzgründer wichtig. Gerade junge Menschen sind dafür prädestiniert, im IT-Bereich neue Unternehmen zu gründen. Daher müssen die von hoher Arbeitslosigkeit betroffenen Länder Existenzgründungen erleichtern und den Zugang zu Risikokapital und zinsgünstigen Krediten ermöglichen.

In einigen Ländern (z. B. Frankreich) ist der gesetzliche Mindestlohn $\mathrm{zu}$ hoch, so dass er besonders für jugendliche Arbeitskräfte zu einem Einstellungshemmnis geworden ist. Daher sollte er dort abgesenkt oder zumindest auf mittlere Frist nicht angehoben werden. 


\subsection{Strukturelle Bedingungen für eine niedrige Jugendarbeitslosigkeit}

Für die Chancen junger Menschen ist zunächst ein leistungsfähiges Schulsystem wichtig. Wie die Qualität eines Bildungssystems im internationalen Vergleich aussieht, kann man z. B. daran ablesen, wie ein Land in PISA-Studien abgeschnitten hat. Wie das Beispiel des häufigen PISA-Siegers Finnland zeigt, schützt ein gutes Testergebnis aber nicht immer vor hoher Jugendarbeitslosigkeit. Wesentlich für die Bewertung der Qualität eines Bildungswesens ist auch, wie viele Schüler ohne Examen die Schule verlassen. Besonders ist darauf zu achten, wie Minderheiten (z. B. Sinti und Roma, Kinder aus Migrantenfamilien) abschneiden. Dazu gehört auch, dass die Schule durch sozial- und jugendpädagogische Maßnahmen begleitet wird, um z. B. Schulschwänzer oder Schulabbrecher noch zu einem qualifizierten Abschluss zu bringen. Wenn solche Personen nach Ende der Schule noch nicht ausbildungsreif sind, müssen sie zumindest wie in Deutschland über ein Übergangssystem (staatliche Förderung durch Praktika, Schule, Betriebswerkstätten) an die Ausbildungsreife herangeführt werden.

Weiterhin ist zu fragen, wie lange die allgemeine Schulbildung dauern soll oder wie viele Schüler die Hochschulreife erwerben und ein Studium aufnehmen. In vielen EU-Ländern gab es eine einseitige Orientierung darauf, durch ein Studium einen gutbezahlten und sicheren Arbeitsplatz im öffentlichen Dienst zu erhalten. Daher gab es zu viele Hochschulabsolventen, aber eine Vernachlässigung mittlerer Qualifikationen. Außerdem können Hochschulen viele Studienplätze in den billigeren Geistes- und Gesellschaftswissenschaften anbieten, die aber schlechtere Berufschancen als Ingenieurwissenschaften haben. Polen ist ein Beispiel für eine zu große Anzahl von Studierenden, die vielfach Fächer besuchen, die an den Erfordernissen des Arbeitsmarktes vorbei gehen. ${ }^{17}$ In Deutschland ist nur ein kleiner Teil der Hochschulabsolventen in einer Tätigkeit beschäftigt, für den kein Hochschulabschluss erforderlich ist. Hingegen ist dieser Anteil z.B. in Spanien besonders hoch.

17 Vgl. M. Polakowski, Youth Unemployment in Poland, op. cit., S. $15 f$. 
Wichtig ist zudem die Etablierung von Ausbildungssystemen, die einen Theorie- und Praxis-Zusammenhang vermitteln. Dies kann durch ein berufliches Ausbildungssystem geschehen, was jedoch kooperative Strukturen zwischen Arbeitgebern und Zusammenschlüsse von Arbeitgebern (Kammern) voraussetzt. Zusammenschlüsse von Unternehmen sind in diesem Kontext notwendig, weil besonders wenn viele kleine Betriebe existieren, eine breite Berufsqualifikation nicht allein in spezialisierten Kleinbetrieben erfolgen kann. Vielmehr muss in diesen Fällen die praktische Ausbildung überbetrieblich ergänzt werden. ${ }^{18}$ Weiterhin ist notwendig, dass der Staat über sein schulisches Bildungssystem die notwendige komplementäre Ausbildung bereitstellt. Dabei dürfen keine systematischen Diskrepanzen auftreten. Wenn in Betrieben eine neue IT-Technik genutzt wird, darf in den Schulen nicht mit einer veralteten Soft- und Hardware gearbeitet werden, weil die notwendigen Mittel zu Anschaffung fehlen oder die Lehrer nicht hinreichend weitergebildet wurden.

Insbesondere die Innovationsfähigkeit von kleineren und mittleren Betrieben hängt in der Schweiz, in Deutschland und Österreich vom dualen Ausbildungswesen ab. Sie sind bedeutend innovativer als vergleichbare Betriebe in Großbritannien oder Frankreich. ${ }^{19}$

Die dafür notwendige Sozialpartnerschaft, die bei allen Beteiligten den Aufbau von gegenseitigen Vertrauen und eine Langfristperspektive erfordert, muss in vielen EU-Staaten wachsen. So wird in Schweden ${ }^{20}$, wo die Jugendarbeitslosigkeit 2014 über 22\% betrug, von Gewerkschaften ein duales Ausbildungssystem abgelehnt, weil die relativ geringen Ausbildungsvergütungen als „Ausbeutung“ eingeordnet wurden, statt als langfristig sinnvolle Investition in das eigene „Humankapital“. So könnte dann die Arbeitsbeziehung insgesamt gerechter ausgebaut werden, so dass nicht nur die Besitzstände von Arbeitsplatzbesitzern geschützt werden, sondern es zu einer solidarischen Verteilung von Arbeit und Einkommen

\footnotetext{
18 Vgl. W. Eichhorst, H. Hinte, U. Rinne, Jugendarbeitslosigkeit in Europa..., op. cit., S. 10.

19 Vgl. F. Rauner, Was tun gegen die Jugendarbeitslosigkeit in Europa? Einführung der dualen Berufsausbildung, „Ifo-Schnelldienst“ 66 (2013) 16, S. 18-21, bes. 18.

$20 \mathrm{Vgl}$. K. Brenke, Jugendarbeitslosigkeit: Bildungsreformen statt Beschäftigungsprogramme, „DIW-Wochenbericht“ 25 v. 17.6.2014.
} 
auch zwischen Generationen kommt. Wenn Ausbildung und Studium zu wesentlichen Teilen in der Praxis erfolgt, gibt es nach dem qualifizierten Abschluss vielfach eine reibungslose Übernahme ohne eine friktionelle Arbeitslosigkeit wie sie nach einem Hochschulstudium oder einer rein schulischen Ausbildung üblich ist. ${ }^{21}$

Nur bei einem langfristigen Denken bei Jugendlichen, Eltern, Unternehmen und Gewerkschaften werden sich die Investitionen in berufliche Bildung durchsetzen. Außerdem muss sich ein qualifizierter Abschluss durch Bezahlung und Aufstiegsmöglichkeiten (Meister, Techniker, Zugang zum Studium ohne Abitur) lohnen. Dies sind Voraussetzungen, die sich auch unter günstigen Bedingungen erst langfristig entwickeln werden. ${ }^{22}$

Im Hochschulbereich ist an duale Studiengänge (Kombination von Praxis und Studium) sowie an eher praxisorientierten Fachhochschulen zu denken, in denen die Dozenten selbst über eine mehrjährige Praxis in Unternehmen, Verwaltungen und Nicht-Regierungs-Organisationen oder gemeinnützigen Betrieben verfügen.

Universitäten haben die Verantwortung, Studiengänge so zu konzipieren, dass Studien in begrenzter Zeit absolvierbar sind. Dazu sind die notwendigen personellen und sachlichen Voraussetzungen auch vom Staat bereitzustellen. Falls es Studienbeiträge bei staatlichen oder privaten Hochschulen gibt, muss durch Stipendien oder zinslose Kredite allen Interessierten ein Studium möglich sein.

Universitäten tragen auch für die Beschäftigungsfähigkeit ihrer Absolventen Verantwortung. Dies hat sich sowohl im Studienangebot wie in Vermittlungs- und Beratungsangeboten (z. B. Praktika) niederzuschlagen.

Eine umstrittene Frage ist ${ }^{23}$, wie genau in einem Land befristete Arbeitsverträge und der Kündigungsschutz für Arbeitnehmer gehandhabt werden. In einigen Ländern ist die Jugendarbeitslosigkeit deshalb besonders hoch, weil im Rahmen von Arbeitsmarktreformen für neue Beschäftigungsverhältnisse befristete Arbeitsverträge erleich-

${ }_{21}$ Vgl. H. Dietrich, Jugendarbeitslosigkeit aus einer europäischen Perspektive, Theoretische Ansätze, empirische Konzepte und ausgewählte Befund, „IAB-Discussion Paper“ 24 (2015), S. 6.

22 Vgl. G. Bosch, Was tun gegen die Jugendarbeitslosigkeit in Europa?, „Ifo-Schnelldienst“ 66 (2013) 16, S. 6-10.

${ }^{23}$ Vgl. W. Eichhorst, H. Hinte, U. Rinne, Jugendarbeitslosigkeit in Europa..., op. cit., S. 12. 
tert wurden, während länger beschäftigte Arbeitnehmer einen hohen Kündigungsschutz genießen.

\section{Schlussbemerkung}

Eine hohe Arbeitslosigkeit Jugendlicher stellt ein erhebliches soziales Übel dar. Wenn die Arbeitslosigkeit von Jugendlichen in einem Land die der Erwachsenen deutlich übertrifft, ist dies ein drastisches Indiz dafür, dass die Generationengerechtigkeit in einem Land verletzt wird. In solchen Fällen sind die gesellschaftlichen Institutionen, z. B. beim Übergang von Schule in den Berufen, nicht so organisiert, dass sie jüngeren Menschen faire Zukunftschancen geben. Es ist daher erforderlich, dass viele EU-Länder vor allem ihr berufliches Ausbildungssystem neu organisieren, um dauerhaft dem Übel der Jugendarbeitslosigkeit abzuhelfen.

Papst Franziskus griff die Frage der Jugendarbeitslosigkeit auf. Junge Leute, die arbeitslos seien, nannte der Papst „»die neuen Ausgegrenzten unserer Zeit«. Stellt euch vor, in einigen Ländern Europas, in diesem unserem Europa, das so gebildet ist, gibt es bis zu $40 \%$ Jugendarbeitslosigkeit, $47 \%$ in anderen Ländern, in anderen $50 \%$. Aber was tut ein junger Mensch, der nicht arbeitet? Wo endet er? In den Abhängigkeiten, in psychischen Krankheiten, im Selbstmord. Und nicht immer werden die Statistiken der Selbstmorde von Jugendlichen veröffentlicht. Das ist eine Tragödie: es ist die Tragödie der neuen Ausgeschlossenen unserer Zeit. Und sie werden ihrer Würde beraubt. Die menschliche Gerechtigkeit erfordert Zugang zur Arbeit für alle." ${ }^{{ }_{24}}$

24 Papst Franziskus, Ansprache für Mitglieder des „Movimento Christiano Lavoratori“ am 16.1.2016, „Osservatore Romano“ (Deutsche Ausgabe) 46. Jg., Nr. 4 v. 29.1.2016, S. 8. 


\section{Summary}

\section{Jugendarbeitslosigkeit - eine große sozialethische Herausforderung in Europa}

Nach der weltweiten Finanzkrise 2007/2008 ist die Jugendarbeitslosigkeit in vielen europäischen Ländern stark gestiegen, während sie in einigen Ländern (Deutschland, Österreich) gering blieb oder gar sank. Ein verpasster Berufseinstieg kann für die Biographie junger Menschen gravierende Konsequenzen haben und ihr Vertrauen in die politische und wirtschaftliche Ordnung herabsetzen. Daher sind kurzfristige Überbrückungsmaßnahmen für die aktuell Betroffenen nötig. Langfristig kommt es zudem in vielen europäischen Ländern mit hoher Jugendarbeitslosigkeit darauf an, durch eine bessere Verzahnung von Ausbildungs- und Beschäftigungssystem den Berufseinstieg der jungen Generation zu gewährleisten.

Schlüsselwörter: Jugendarbeitslosigkeit, Generationengerechtigkeit, Europa,

Berufsausbildung, Finanzkrise

\section{Youth unemployment - a central social ethical challenge in Europe}

After the global financial crisis 2007/2008 youth unemployment in many European countries has risen sharply, while in some countries (Germany, Austria) remained low or even decreased. A missed Career entry can for the biography of young people have serious consequences and reduce their confidence in the political and economic order. Therefore, short-term stopgap measures are necessary for the currently affected. In many European countries with high youth unemployment important it is also important to ensure the Career entry of the young generation through a better integration of training and employment system.

Keywords: Youth unemployment, Intergenerational justice, Europe, vocational training, financial crisis

\section{Bibliografie}

Brenke K., Jugendarbeitslosigkeit: Bildungsreformen statt Beschäftigungsprogramme, „DIW-Wochenbericht“ 25 (2014) vom 17.6.2014.

Bosch G., Was tun gegen die Jugendarbeitslosigkeit in Europa, "Ifo-Schnelldienst" 66 (2013) 16, S. 6-10.

Bosch G., Jugendarbeitslosigkeit in Europa - warum versagen milliardenschwere Hilfsprogramme, „Ifo-Schnelldienst“ 68 (2015) 17, S. 7-11.

Dietrich H., Jugendarbeitslosigkeit aus einer europäischen Perspektive. Theoretische Ansätze, empirische Konzepte und ausgewählte Befund, „IAB-Discussion Paper” 24 (2015). 
Eichhorst W., Hinte H., Rinne U., Jugendarbeitslosigkeit in Europa: Status Quo und (keine?) Perspektiven, IZA Standpunkte Nr. 57, Bonn.

Möller J., Jugendarbeitslosigkeit in Europa: Generation ohne Zukunft, ,Ifo-Schnelldienst “ 68 (2015) 17, S. 3-6.

Papst Franziskus, Ansprache für Mitglieder des „Movimento Christiano Lavoratori“ am 16.1.2016, „''Osservatore Romano“ (Deutsche Ausgabe) 46. Jg., Nr. 4 v. 29.1.2016, S. 8.

Polakowski M., Youth Unemployment in Poland, Bonn 2012 (Friedrich-Ebert-Stiftung).

Rauner F., Was tun gegen die Jugendarbeitslosigkeit in Europa? Einführung der dualen Berufsausbildung, „Ifo-Schnelldienst“ 66 (2013) 16, S. 18-21.

Sohn K. D., Czuratis S., Jugendarbeitslosigkeit in den Mitgliedstaaten der EU - was kann und sollte die EU tun, „Ifo-Schnelldienst“ 66 (2013) 16, S. 14-17.

Tamesberger D., Jugendarbeitslosigkeit in Europa: Eine Beschreibung des Problemausmaßes und der Folgen, „Wiso“ 1 (2014), S. 139-158.

Wiemeyer J., Die Solidarischen Verpflichtungen der älteren Generation gegenüber Kindern und Jugendlichen, [in:] Intergenerationalität zwischen Solidarität und Gerechtigkeit, Hg. J. Eurich, P. Dabrock, W. Maaser, Heidelberg 2008, S. 133-147.

Wiemeyer J., Gerechtigkeit zwischen Generationen als wirtschaftsethisches Problem, „Ethica“ 12 (2004) 1, S. 71-94.

Wiemeyer J., Sprawiedliwość międzypokoleniowa jako wyzwanie społeczno-etyczne, „Roczniki Nauk Społecznych“ 3 (2011) 39, S. 69-87.

Wiemeyer J., Alterssicherung und Politik bei schrumpfender Bevölkerung, „Polonia Sacra" 19 (2015) 1 (38), S. 5-26. 